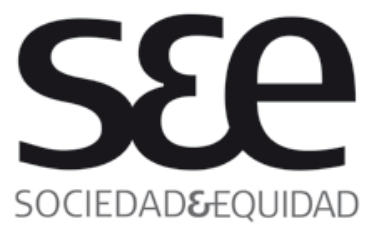

SOCIEDADEEQUIDAD

\section{Estudiar un postgrado en el extranjero: ¿una migración previsible?}

Postgraduate studies abroad: ¿A forseeable migration?

\title{
Resumen
}

La presente contribución pretende incorporar la dimensión subjetiva al estudio de la migración de estudiantes para comprender la manera en que ciertos jóvenes profesionales latinoamericanos deciden realizar estudios de postgrado en el extranjero. Consecuentemente, el énfasis está puesto en la exploración de los procesos que contribuyen a esta migración en el país de origen desde el punto de vista de los suj etos migrantes. Dos preguntas guían nuestra reflexión: ¿Cuáles son las dinámicas sociales y profesionales que incitan a realizar estudios de postgrado en el extranjero? ¿Qué motivaciones y aspiraciones personales tienen los individuos para embarcarse en dicho proyecto migratorio? Para proponer elementos de respuesta, esta investigación se basa en 63 entrevistas biográficas y comprensivas efectuadas entre 2009 y 2011 a jóvenes profesionales, de nacionalidad chilena o colombiana, al término de sus estudios de máster o doctorado en las ciudades de París, Nueva York o Boston.

\section{Palabras Claves}

Migración altamente calificada, Postgrado, Trayectoria social, Trayectoria profesional, Bifurcación.

\footnotetext{
${ }^{1}$ La autora es Doctora en Sociología. Sus líneas investigativas son: migraciones, movilidad social, sociología de las élites, poblaciones de alta calificación.
} 


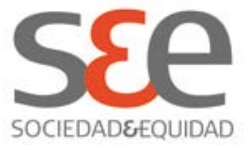

\begin{abstract}
This contribution aims to incorporate a subjective dimension to the study of students migration in order to understand how some young professionals decide to pursue graduate studies abroad. Consequently, we emphasize the exploration of processes that contribute to the migration from the country of origin from the subjetcts point of view. Two questions guide our reflexion: What are the social and professional dynamics that encourage graduate study abroad? What are the individual's motivations and aspirations to start this migration project? This research is based on 63 biographical and comprehensive interviews made between 2009 and 2011 of young Chilean and Colombian professionals, following their master or doctoral studies in the cities of Paris, New York or Boston.
\end{abstract}

\title{
Keywords
}

Highly skilled migration, Graduate students, Social trajectory, Professional career, Bifurcation.

La globalización de la actividad económica tiene efectos concretos para las poblaciones de alta calificación. La reestructuración de los mercados de trabajo les exige el desarrollo de destrezas internacionales para ponerlas al servicio de las empresas y participar de los intercambios entre diferentes ciudades, países y regiones del mundo (Sassen, 2001; Wagner, 2005). El dominio de idiomas, así como de ciertos contenidos estandarizados y el prestigio de adquirir un perfil "internacional" son un atractivo innegable para conseguir un trabajo calificado. Los sistemas de educación superior de los países desarrollados juegan a su vez la carta de la movilidad internacional, bajo una lógica de competencia y privatización. Las universidades buscan atraer a los estudiantes extranjeros para aumentar el número de matrículas y procurarse así recursos financieros adicionales (Bolzman y Guissé, 2010: 142). Finalmente, en los países periféricos, la alta valoración de los expertos formados en el hemisferio Norte resulta una manera de aceptar y reproducir un cierto orden geopolítico entre países.

En este marco, la migración de estudiantes hacia un grupo limitado de países desarrollados forma parte de la reciente aceleración y diversificación de los flujos migratorios internacionales. De acuerdo a Catherine Wihtol de 


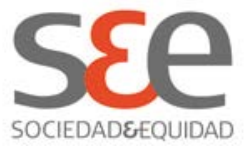

Wenden, esta extensión se origina, entre otros, en la articulación entre los efectos del envejecimiento demográfico en las regiones tradicionales de acogida y la incitación a partir existente entre las poblaciones jóvenes, urbanas y cada vez más educadas de las regiones periféricas (Wihtol de Wenden, 2010: 43). Conviene considerar también como parte de este contexto, las inversiones crecientes de los países en vías de desarrollo en el llamado capital humano avanzado. Estos esfuerzos, mayoritariamente públicos, resultan indiscutibles en el contexto latinoamericano si consideramos los recursos destinados por los gobiernos a programas de becas de estudios o de investigación y tecnología. Adoptando el modelo del brain gain, las inversiones de los países del Sur tienden a combinar la migración de estudiantes y profesionales para su perfeccionamiento en el extranjero con el reforzamiento de sus propias instituciones académicas y de investigación para luego estimular su regreso e instalación (Meyer, 2008: 40).

Estudiar estas prácticas migratorias únicamente desde un punto de vista macrosocial resulta insuficiente. Este enfoque permite identificar por qué ciertas poblaciones tienen una propensión más fuerte que otras a emigrar, sin embargo, no es posible dilucidar por qué ciertos individuos (y no otros) parten en un momento determinado. La presente contribución pretende incorporar la dimensión subjetiva al estudio de la migración de estudiantes de postgrado para comprender la manera en que algunos jóvenes profesionales latinoamericanos deciden realizar estudios de especialización en el extranjero. Consecuentemente, el énfasis está puesto en la exploración de los procesos que contribuyen a esta migración en el país de origen desde el punto de vista de los sujetos migrantes. Dos preguntas guían nuestra reflexión: ¿Cuáles son las dinámicas sociales y profesionales que incitan a realizar estudios de postgrado en el extranjero? ¿Qué motivaciones y aspiraciones personales tienen los individuos para embarcarse en dicho proyecto migratorio? Para proponer elementos de respuesta, esta investigación se basa en 63 entrevistas biográficas y comprensivas efectuadas entre 2009 y 2011 a jóvenes profesionales, de nacionalidad chilena o colombiana, al término de sus estudios de máster o doctorado efectuados las ciudades de París, Nueva York o Boston.

El artículo se organiza en dos partes. La primera explica la construcción teórica del objeto de estudio. Aquí insistimos sobre el carácter migratorio de la práctica de estudiar un postgrado en el extranjero para luego inscribir nuestra investigación en el campo de estudio de las trayectorias sociales. Desde esta perspectiva, nos preguntamos por aquello que resulta más o menos previsible en términos de un itinerario social para examinar la articulación entre agencia y determinismo. La segunda parte del artículo presenta una parte del análisis empírico de las entrevistas efectuadas a jóvenes profesionales en movilidad. Focalizándonos en el estudio del período entre la obtención de su pregrado y el 


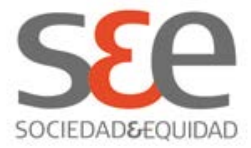

momento de la emigración, proponemos una tipología para explicar cómo la experiencia laboral (o su ausencia) en el país de origen contribuye a la elaboración del proyecto migratorio. La conclusión del artículo sintetiza los aspectos más o menos previsibles de las trayectorias estudiadas tomando en consideración la perspectiva subjetiva de los migrantes y los cambios estructurales que inciden en ella.

\section{Migraciones de alta calificación: ¿trayectorias previsibles?}

Estudiar un postgrado en el extranjero se constituye como una práctica migratoria. Ya en los años ochenta, Hervé Domenach y Michel Picouet insisten sobre la necesidad de clasificar los desplazamientos humanos de manera de abarcar prácticas de movilidad más o menos temporales. Ellos proponen la noción de espacio de vida que designa todos los espacios, físicos y cognitivos, en los que el ser humano evoluciona. A partir de este término, los autores definen la migración como todos aquellos desplazamientos que modifican los espacios de vida de un individuo, sea por una suma o reducción de aquéllos, o bien por un cambio radical y/ o permanente en su interior (Domenach y Picouet, 1987: 9-10). En la actualidad, los estudios migratorios se han vuelto progresivamente receptivos al análisis de prácticas migratorias que no implican necesariamente una ruptura con el país de origen ni tampoco una inserción definitiva en otro lugar (Cortes y Faret, 2009: 11). En esta línea, el hecho de vivir en otro país con el objetivo de estudiar durante al menos un año implica que las personas agregan nuevos repertorios a sus espacios de vida de manera más o menos definitiva. Por otra parte, desde el punto de vista del individuo, el cotidiano de los estudiantes extranjeros se estructura de manera similar que para otros migrantes en los países desarrollados. Catherine Agulhon y Angela Xavier de Brito afirman que ellos comparten con otras poblaciones móviles lo que ellas denominan el habitus del migrante. Su investigación cualitativa sobre los estudiantes extranjeros en París da cuenta de sentimientos de dominación e inferioridad en la sociedad de acogida, así como de situaciones económicas difíciles. Estas condiciones explican, entre otras, el relativo aislamiento entre estudiantes extranjeros y que resulta una característica común de varias experiencias migratorias (Agulhon y Xavier de Brito, 2010: 19).

Desde un punto de vista teórico, la decisión migratoria no se reduce a una sola causa ni a la dimensión profesional de una determinada trayectoria social. El proyecto de estudiar en el extranjero tiene relación con la manera en que los individuos imaginan y conciben su desarrollo profesional a partir del ejercicio de una disciplina y su mercado de trabajo. Esta representación es variable, pero se construye en estrecha relación con los aspectos biográficos y familiares del individuo, así como con su posición social. Estas "predisposiciones" en términos de Pierre Bourdieu existentes a nivel micro 


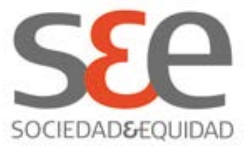

social se combinan con circunstancias macro sociales que pueden resultar más 0 menos favorables para el proyecto de estudiar postgrado en otro país (como por ejemplo, una crisis económica o la disponibilidad de becas para realizar dichos estudios). La articulación entre un nivel de análisis micro y macrosocial puede ser abordada utilizando algunos conceptos de la literatura sobre las trayectorias sociales.

La noción de trayectoria social intenta abordar la relación entre acción y determinismo desde un punto de vista algo diferente. Buena parte de la sociología contemporánea otorga una gran importancia a los efectos de la historia familiar y del nivel educacional de los padres sobre el individuo, así como de las múltiples disposiciones culturales y sociales que se adquieren durante la infancia. Desde esta visión, las acciones de los individuos quedan determinadas a su capacidad de re-apropiación o de re-interpretación de las herencias sociales. Los estudios que se interesan en la noción de trayectoria desplazan el foco de la interpretación hacia lo que resulta en menor o mayor medida discontinuo o imprevisible en un itinerario social.

En este marco, nuestra investigación se inspira del modelo bifurcativo elaborado por Frédéric De Coninck y Francis Godard. Este modelo afirma que "cada existencia se divide en secciones tranquilas donde las cosas siguen su curso, y en momentos decisivos donde todo entra en juego y los destinos bifurcan unos de otros" (De Coninck y Godard, 1990: 36). Esto significa que frente a un evento biográfico - en este caso, la decisión de hacer un postgrado en el extranjero - la interpretación busca diferenciar las causas accidentales de aquéllas que pueden ser consideradas como predeterminadas. Por ejemplo, podríamos pensar que la práctica estudiada es más probable en el caso los científicos 0 de trabajadores que deben perfeccionarse para conservar su empleo.

Por otra parte, decidimos basarnos también en la contribución de Valentine Hélardot que distingue, entre otros, dos tipos de bifurcaciones en el ámbito de las trayectorias profesionales (Hélardot, 2010: 164-166). El primer tipo se trata de la "bifurcación activa" que designa a las personas que deciden en un momento dado romper con la situación estable en la que viven, tomando así la iniciativa del cambio que se desarrolla luego en su trayectoria. Este tipo de bifurcación podría representar, por ejemplo, la situación de algunos profesionales que han adquirido una estabilidad económica y profesional en su país y que deciden poner fin a este período para volver a ser estudiantes por un tiempo. El segundo tipo que retenemos es la bifurcación tipo "concurso afortunado de circunstancias" que describe un cambio en la trayectoria laboral considerado beneficioso por el individuo a pesar de que no se produce inicialmente gracias a su acción. Este cambio se produce bajo la influencia de 


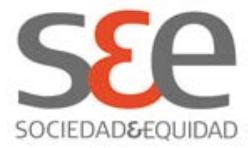

un contexto externo favorable para a los proyectos de la persona. Este tipo de bifurcación puede ilustrar, por ejemplo, la situación de algunos jóvenes investigadores que parten a estudiar motivados por la obtención de una beca en la universidad donde trabajan.

En último término, la decisión migratoria es resultado de fuerza de la acción individual y la influencia de un cierto contexto, sin embargo, ella da cuenta también de un tercer elemento. En toda trayectoria es posible observar la acción de un gatillador o evento particular que provoca la puesta en marcha efectiva del proyecto migratorio. Este elemento puede ser, por ejemplo, el término de un contrato de trabajo, una ruptura amorosa, un accidente en la familia, o cualquier otra circunstancia que lleve al individuo a reflexionar y cuestionar en cierta medida la inercia de su trayectoria. En términos teóricos, la idea del gatillador permite explicar por qué finalmente no se produce un número mayor de salidas que coincide con todas las personas que viven en las mismas circunstancias estructurales y tienen herencias familiares similares.

\section{Las trayectorias profesionales en el país de origen: una tipología para comprender por qué y cómo los jóvenes profesionales deciden estudiar un postgrado en el extranjero}

El análisis de la experiencia laboral previa, o la falta de ella en algunos casos, ofrece pistas sólidas para comprender las razones por la cuales los jóvenes profesionales deciden emigrar en lugar de seguir en su país sea trabajando o estudiando. Estas experiencias fueron clasificadas en seis grupos diferentes ${ }^{2}$. Cada uno ilustra una trayectoria profesional particular que permite comprender cómo y con qué objetivos los individuos deciden partir al exterior para especializarse. El primer tipo considera sólo aquellos entrevistados que no tienen experiencia laboral y que parten al extranjero sin antes haber buscado un trabajo. El resto de los encuestados cuenta entre uno y diez años de experiencia profesional antes de partir. Distribuyéndose entre cinco tipos de trayectorias, cada una de estas categorías reúne a profesionales que persiguen obj etivos diferentes de acuerdo a su lugar de trabajo particular y experiencia.

Los jóvenes sin experiencia laboral: continuar estudiando en el extranjero

Cinco entrevistados partieron al extranjero con el objetivo de especializarse inmediatamente después de haber terminado su pregrado en Chile o en Colombia. Estos relatos dan cuenta de una gran heterogeneidad de

2 Este ejercicio se inspira del método del tipo ideal de Marx Weber donde cada categoría o tipo identificado es una construcción simplificada de la realidad y cuyo objetivo fundamental no es otro que contribuir a la comprensión sociológica (Schnapper, 1999: 2). 


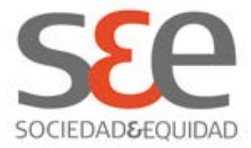

situaciones, sin embargo, un elemento común parece imponerse: la decisión de partir resulta poco previsible considerando sus experiencias anteriores en términos escolares, familiares e individuales. Su decisión de emigrar se concretiza gracias a diversas oportunidades que se presentan en el momento. Veamos tres ej emplos.

Antonio es un economista chileno quien, al momento de la entrevista, sigue un doctorado en París. Él se fue a vivir a Francia a la edad de veintitrés años luego de obtener su título profesional. Desde el punto de vista de su biografía, su partida resulta inesperada pues no tenía una historia familiar o personal que lo vinculara con ese país. Durante sus estudios, Antonio vive con sus padres, un contador y una profesora universitaria de música, en un barrio acomodado de Santiago. Casi al término del programa, su padre falleció y poco después, Antonio decide realizar un intercambio universitario en España. "Cuando vivía en Barcelona, me juntaba con muchos franceses y así fue como conocí a mi novia. Cuando terminó ese año, volví para dar mi examen de grado y después me fui a vivir con ella a Lyon". La pareja decide casarse y Antonio se matricula en una universidad francesa. "No sé por qué en realidad. Me podría haber devuelto, o podría haber estado un rato y haberme devuelto después. No fue tampoco porque quería viajar porque ya conocía bien Europa, había paseado harto cuando viví en Barcelona. Pero decidí quedarme. Y aunque no hablaba nada de francés, dije voy a intentar meterme en un máster pensando en que quizás me podría servir después". La estrategia de estudiar permite conocer nuevas personas, aprender el idioma y vincularse progresivamente al mundo social y laboral del país de acogida. Una vez terminado su programa de magíster de dos años, las buenas calificaciones de Antonio y su interés por el mundo académico lo llevan a comenzar un contrato de ayudante y una tesis doctoral en economía en París.

Otro caso es el de Alicia, una arquitecta colombiana que realiza una pasantía profesional en París gracias al contacto con profesionales expatriados de su país en Francia. En esta oportunidad, el recurso que permite la emigración es el contacto con redes transnacionales a través de su universidad. De la misma manera que Antonio, nada en la biografía de la joven profesional podía presagiar su partida a Francia. Alicia estudió arquitectura en una prestigiosa universidad de Bogotá. Poco después de su graduación, la joven respondió al anuncio de una agencia parisina de arquitectura para hacer una práctica profesional remunerada. Alicia no habla francés, pero ello no es un obstáculo para su selección. El dueño de la empresa era un arquitecto colombiano que estudió en su misma universidad, de manera tal que los equipos de trabajo son bilingües. "La idea era tener una experiencia internacional en mi hoja de vida, aprovechar de viajar y conocer Europa, quizás quedarme varios años estudiando y trabajando, aprender todo lo posible. No 


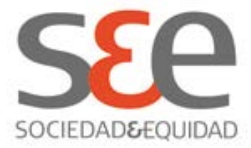

sé, cuando vives en el extranjero, la gente en Colombia te mira después con otros ojos, estás como a otro nivel". En este caso, el proyecto de estudiar al exterior nace más claramente como una estrategia de movilidad social orientada a acelerar la carrera profesional. Sin tener ahorros, Alicia cuenta con el apoyo financiero de sus padres quienes costean sus pasajes y los gastos de su inscripción en la universidad la que resulta indispensable para obtener una visa de estudiante y firmar su contrato.

El tercer ejemplo es un poco diferente a los primeros, debido a que la trayectoria de Jorge -físico chileno que sigue un post-doctorado en Boston- da cuenta de una intensa socialización "internacional" durante su infancia y juventud. Este joven científico pertenece a una familia acomodada de la capital que costeó sus estudios primarios en una escuela bilingüe y luego, un intercambio escolar en Estados Unidos. J orge cursa sus estudios universitarios de pregrado en la Universidad Católica. Sus sobresalientes resultados le permiten desempeñarse como ayudante y cultivar relaciones cercanas con profesores. Al terminar sus estudios, sus mentores lo animan a postular a un doctorado en una universidad norteamericana. "Yo no vengo de una familia de matemáticos, por lo que no sabía muy bien a lo que iba pero pronto entendí que si uno quiere hacer carrera tiene que hacer un doctorado (Pregunta: ¿Y por qué no pensaste por ejemplo en hacer ese doctorado en Chile?) En Chile no hay una amplia gama de estudios de física, así que no tenía otra opción, de hecho, yo me tuve que ir". Dos temas son interesantes en el caso de J orge. Primero, su certeza de "no tener otra opción" permite hacer el nexo con la teoría de la de fuga de cerebros. Frente a la fragilidad de las condiciones para el desarrollo de la investigación de los países periféricos, los científicos se ven en cierta medida forzados a partir hacia los centros especializados de los Estados Unidos. Segundo, el apoyo institucional que posibilita esta migración temprana sin tener ningún otro vínculo ni solicitar recursos económicos adicionales. Gracias al apoyo de sus profesores, J orge obtiene un financiamiento completo para su doctorado en la Universidad de Notredame que pertenece a la red de las universidades católicas entre las cuales existen múltiples convenios.

Para los profesionales que estudian un postgrado en el extranjero sin tener una experiencia laboral previa, la decisión de partir es en cierta medida espontánea y resulta de la articulación de oportunidades que se presentan en el momento. Los jóvenes se afirman en recursos diversos tales como el apoyo familiar o de la pareja, las redes entre universidades y profesores, o los contactos profesionales ligados a una comunidad transnacional. Se trata de individuos que forman parte de clases medias privilegiadas en el países de origen, todos hijos de padres con estudios superiores. Sin embargo estas familias no pertenecen exactamente a los círculos sociales dominantes donde los itinerarios profesionales y sociales esperados dejan poco lugar a la 


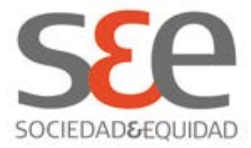

improvisación. En último término, conviene insistir sobre la idea que para este grupo de jóvenes profesionales, el hecho de partir a perfeccionarse en el extranjero es acontecimiento imprevisible y una bifurcación. Prueba de ello es la rapidez con la que estos proyectos se implementaron, en pocos meses de preparación y gracias a la aparición relativamente fortuita de oportunidades favorables del momento. Estas salidas menos previsibles prefieren a Francia como país de destino considerando que allí los programas de estudios de postgrado son menos caros que en Estados Unidos.

Los jóvenes trabajadores de las universidades y la investigación: partir estudiar al extranjero para acelerar la carrera académica o abandonarla.

Casi un tercio de los entrevistados indica haber trabajado como estudiante en su universidad durante época universitaria. Buena parte de ellos, luego de obtener su pregrado, continúa vinculado a su casa de estudios obteniendo un contrato de trabajo como asistente de investigación o en la docencia. De esta manera, algunos jóvenes comienzan tempranamente una carrera profesional en el mundo académico y de investigación. Este tipo de trayectoria abarca diversas disciplinas de estudio. Entre las ciencias duras, tenemos, por ejemplo, el caso de tres ingenieros que luego de obtener su título trabajaron en proyectos de investigación en química, física y procesos industriales. Dentro de las ciencias sociales, varios estudiantes en economía, psicología y sociología también tuvieron sus primeros trabajos con relación a sus profesores y proyectos diversos de su facultad.

El mundo académico como lugar de trabajo valora fuertemente los trabajadores que poseen un grado de máster y doctorado, más aún si éstos son obtenidos en el extranjero. Ello permite a las universidades locales de posicionarse mejor dentro del paisaje globalizado de los sistemas de educación superior en competencia. De esta manera, los jóvenes que comienzan a trabajar allí son estimulados por este medio a elaborar un proyecto de estudios de postgrado en el extranjero para mejorar sus posibilidades de consolidarse en la jerarquía universitaria.

Los relatos recogidos nos permiten reflexionar sobre las condiciones de trabajo en las universidades chilenas y colombianas. Frecuentemente este tipo de experiencia profesional implica una fuerte inestabilidad y bajos salarios. Una manera de retener a los profesionales jóvenes es proponerles una beca para cursar un programa de máster en su misma casa de estudios ${ }^{3}$. Sin tener

3 El costo de este tipo de programa de postgrado suele ser muy elevado en los dos países estudiados. Veamos por ejemplo el costo de dos programas seguidos por algunos de nuestros entrevistados. En la Universidad Católica de Chile, el costo anual del programa de Magíster en Sociología asciende a aproximadamente $€ 5.500$ (3.740.000 


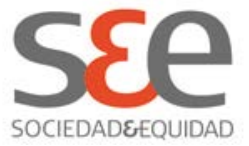

otras alternativas a la mano y frente la posibilidad del desempleo, muchos aceptan esta oferta y fortalecen así sus vínculos con la universidad. En su momento, esta red de contactos resulta altamente efectiva para poner en marcha el proyecto de estudios doctorales en el exterior.

Veamos el ejemplo de César, ingeniero colombiano quien, al momento de la entrevista, cursa los últimos meses de su programa de doctorado en aplicaciones industriales en París. César viene de una familia de clase media, sus padres no hicieron estudios universitarios. "Cuando terminé mis estudios en ingeniería en la (Universidad) Nacional y tuve una oportunidad muy interesante en otra universidad colombiana, en la de los Andes. Un profesor me pidió que hiciera la maestría con él y que al mismo tiempo trabajara en un proyecto de investigación. Puse todo en la balanza: esto de seguir estudiando en la Universidad de los Andes, o dedicarme a buscar cómo partir de inmediato al extranjero. Aunque empecé a estudiar francés, todavía no había elegido un programa, no tenía nada claro en realidad. Y esta oportunidad me permitió continuar estudiando en lugar de parar y no hacer nada. Así fue como me encontré mi tema de estudio". César comienza a trabajar en la universidad como asistente y después del primer año, conoce a su actual director de tesis que enseñaba en uno de los cursos avanzados del programa de maestría.

Luego de dos o tres años, pocos profesionales logran un empleo estable y bien remunerado en el sector universitario. Cuando éste es el caso, los jóvenes quitan rápidamente el domicilio de sus padres y ocupan este puesto de trabajo hasta el momento en que parten al extranjero. Si no es el caso, los jóvenes raramente logran independizarse y deben continuar viviendo con sus padres. Para ellos, la vida laboral se construye como un período de continuidad con la época universitaria que contiene un cierto grado de amargura. Pasado algunos años, el proyecto de estudios en el extranjero parece la mejor oportunidad para salir de esta situación de precariedad y utilizar los recursos profesionales acumulados hasta entonces. Éste es el caso, por ejemplo, de Santiago, chileno, quien al momento de la entrevista es estudiante de doctorado en sociología en Francia. Hijo de un padre periodista y una madre enfermera, Santiago decide estudiar sociología en la Universidad de Chile. Su idea de partir a estudiar en Francia comienza a configurarse con el descubrimiento de ciertos autores franceses. "A pesar de que tenía buenas notas en la Universidad, me costó encontrar trabajo. Traté de hacer algunos proyectos académicos con colegas de la universidad, pero no funcionó porque nos pedían tener un doctorado. En ese momento, yo acababa de terminar una relación y sentía que no tenía lazos con Chile. Al mismo tiempo, no podía hacer

pesos chilenos). En la Universidad de Los Andes, el costo del programa de Maestría en Ingeniería se eleva a aproximadamente $€ 4.880$ (11.240.000 pesos colombianos). 


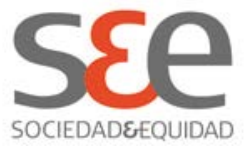

las cosas que quería hacer por falta de experiencia y currículum. Trabajé en varios proyectos, pero nada muy estable. Empecé a privilegiar contratos de investigación, aunque fueran cortos, para tener un mejor currículo para las becas". Santiago vive con sus padres hasta que obtiene una beca para hacer un doctorado en Francia. Su pequeño estudio en una residencia universitaria parisina es su primera casa como joven adulto independiente.

La experiencia de trabajar en las universidades favorece ampliamente el proyecto de estudios en el extranjero. Las redes profesionales creadas participan de manera efectiva durante la fase de preparación del proyecto. Estos contactos son capaces de proporcionar cartas de recomendación y todo "know-how" de los investigadores para preparar sus candidatos a los concursos de becas de estudios. La gran mayoría de este grupo decide estudiar un programa de doctorado para luego volver a trabajar en las universidades locales. Aquéllos que dejado el mundo académico algo decepcionados, deciden al contrario, hacer un programa de máster para continuar una carrera en el sector público o en las empresas. El país de destino parece ser elegido de acuerdo a las redes académicas cultivadas y los convenios entre universidades.

Los trabajadores del sector público: hacer un postgrado en Estados Unidos para ser parte de los policy- makers

Este tipo de trayectoria profesional reúne a jóvenes que han trabajado en el sector público antes de estudiar en el extranjero. Para simplificar, también consideramos en esta categoría a las personas que ocuparon un puesto en una organización internacional y en organizaciones no gubernamentales. Se trata de jóvenes que han estudiado disciplinas que conducen directamente al Estado, como la economía, el derecho o la ciencia política, así como también otras carreras menos específicas, pero afines a la administración pública, como la sociología, la psicología, la historia e incluso el arte. Estos profesionales deciden trabajar inmediatamente después de la entrega de su título universitario y obtienen un trabajo en este sector gracias a sus redes sociales y profesionales. Algunos son reclutados después de una recomendación positiva de la parte de un profesor con experiencia, otros gracias al contacto de un antiguo compañero de la universidad. Ciertos jóvenes son invitados desde el primer día a integrar un puesto gracias a su compromiso con algunos partidos políticos durante la época universitaria.

La inserción laboral de los jóvenes de clases medias recientes se diferencia claramente de aquéllos que provienen de familias adineradas y mejor posicionadas. Estos últimos obtienen su primer empleo a través de redes sociales no profesionales que los identifican eficazmente como miembros de las clases dominantes y reflejan su participación en las estructuras públicas. Algunas de estas redes son la familia extensa, los grupos religiosos o los padres 


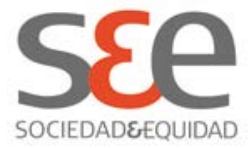

de los amigos de la escuela. Para ellos, la idea de perfeccionarse en el extranjero es un proyecto antiguo, deseado e indispensable para acceder a las más altas posiciones profesionales y sociales.

Para los entrevistados que pertenecen a una clase media reciente y que han tenido pocas experiencias internacionales (aprendizaje de idiomas, intercambios escolares, viajes, etc.), el proyecto de estudiar al extranjero se inicia durante la experiencia laboral en el sector público. Estas instituciones los alientan a seguir estudiando y procuran modelos cercanos a imitar de profesionales que se perfeccionan en el exterior. Su curiosidad por viajar y descubrir otras culturas se despierta al mismo tiempo que la ambición profesional. Por otro lado, el trabajo en el sector público provee los medios económicos necesarios para tomar vacaciones por primera vez, ahorrar o costear un curso de idioma. Los jóvenes adquieren así de manera acelerada las competencias cognitivas y financieras necesarias para realizar un postgrado en el extranjero.

La disciplina parece también jugar un rol importante en el éxito profesional de las trayectorias en el sector público. Algunos graduados en economía o derecho comienzan una rápida carrera ascendente en la jerarquía pública y ocupan en el corto plazo altos puestos de responsabilidad. La gran mayoría de los entrevistados ocupan puestos profesionales estables menos elevados, pero que les permiten construir su independencia económica.

En este tipo de trayectoria, el proyecto de perfeccionarse en el exterior parece íntimamente ligado a la dinámica interna de las estructuras públicas. Una especialización internacional es necesaria para subir en la jerarquía del Estado. Así lo afirma Carmen, economista colombiana que estudia un máster en Políticas Públicas en la Universidad de Columbia en Nueva York. "En mi trabajo, todos los miembros del equipo del presidente Uribe había estudiado en Estados Unidos. Es como una ola, tú ves cómo la gente se prepara para salir, luego se va mientras que otros vuelven a ocupar un puesto de mayor responsabilidad. $Y$ en un momento dado, es tu turno de partir". Estos profesionales son los policymakers que cursan un postgrado en las más prestigiosas universidades de Estados Unidos financiados por programas como Fulbright, que buscan favorecer las transferencias técnicas e ideológicas entre gobiernos. El proyecto de estudiar en el exterior articula convenientemente el deseo de movilidad profesional de los trabajadores del sector público y la dinámica de las estructuras públicas particularmente ávidas de profesionales y técnicos de alto nivel de rango internacional. 


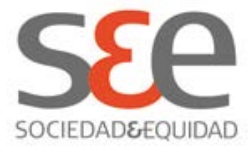

Los trabajadores del sector privado: en la búsqueda de título "internacional"

Menos de diez entrevistados de la muestra componen esta categoría caracterizándose por una fuerte homogeneidad entre sus miembros. Incluimos aquí a profesionales que han obtenido un título en ingeniería o en gestión empresarial y que trabajan luego bancos u otras empresas internacionales. Este entorno de trabajo, globalizado e internacional, favorece y valora particularmente un perfil internacional. Veamos a la historia de Bruno, un ingeniero chileno que a sus 31 años decide renunciar su trabajo para hacer un máster en Estados Unidos. Hijo de un padre ingeniero y una madre profesora, Bruno pertenece a una familia acomodada. Su hermano mayor realizó también estudios de postgrado en ese país. "Después de estudiar ingeniería en la Católica, me fui a trabajar a " $X$ " que es una compañía internacional de telecomunicaciones. (...) Hasta ese momento, yo estaba seguro de que me encantaban las ciencias puras y que si me alejaba de ellas me iba a frustrar. Pero en " $X$ " tuve la oportunidad de trabajar en cosas súper operativas, en la gestión, y me encantó poder hacer una contribución más potente que entregar unos cálculos perfectos. Me ascendieron rápidamente y me sentía super valorado en la empresa. Desde una perspectiva personal, ese trabajo me permitió ser independiente e irme de la casa de mis papás (...) Quería ir a estudiar afuera hace mucho tiempo. Después de mi viaje (como turista) a Berlín, o incluso cuando hice un intercambio en California en mi último año en la (Universidad) Católica, la idea de vivir en el extranjero, de conocer otros lugares, siempre la tuve. No lo hice antes porque quería hacer otras cosas primero, vivir solo por ejemplo. Después del tercer año en la empresa retomé mi proyecto y me puse preparar los exámenes y el GMAT para postular al año siguiente". Para los jóvenes pertenecientes a familias acomodadas, el proyecto de perfeccionarse en el exterior es una oportunidad para utilizar y actualizar sus inclinaciones cosmopolitas adquiridas progresivamente (Wagner, 2007: 4-7) como el paso por un colegio bilingüe o los viajes familiares. Estudiar afuera no es considerado como una experiencia definitiva, sino como un período breve e intenso enfocado a adquirir competencias específicas sin alejarse demasiado tiempo del ejercicio profesional y las exigencias de las empresas. Al mismo tiempo, la práctica responde a una estrategia de ascensión profesional. Así lo explica Julián, economista colombiano que sigue una especialización de máster en Nueva York quien trabaja antes en un banco durante tres años. "En las empresas multinacionales, los que tienen una maestría suben más rápido, ésa es la vía a seguir". Considerar esta práctica de manera más práctica resulta característico de los profesionales de clases medias. Para ellos, el futuro laboral es más incierto debido a que poseen, en palabras de Bourdieu (AÑO), a un capital social menos sólido. 


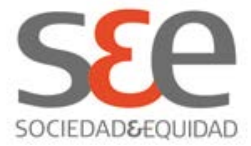

Los discursos coinciden en una idea fundamental: partir a estudiar es la oportunidad de romper con una cierta estabilidad y monotonía laboral. Al cabo de algunos años en las empresas, los profesionales logran una experiencia profesional importante y salarios satisfactorios. Las alternativas evaluadas en ese momento son especializarse en el exterior o bien, dejar su trabajo actual para buscar otro más interesante. La decisión de retomar los estudios no es improvisada, ella da cuenta de una reflexión sistemática que se transforma en un plan coherente a nivel profesional por el que vale la pena dejar la estabilidad ganada. En cuanto al tipo de formación, los programas seleccionados son orientados al trabajo, es decir, una especializada de máximo dos años a nivel de máster. Volviendo al relato de Bruno: "Yo estaba entre hacer un MBA o un máster orientado a la investigación. Al final me decidí por un programa entre los dos, entre ciencia y administración (...) Cuando me enteré de que fui aceptado, postulé a BECAS CHILE. Yo soy de los que no deberían haber sido financiado por las políticas públicas, porque estaba dispuesto a gastar todos mis ahorros y endeudarme para venir aquí. Lo tenía todo preparado hace años. (Pregunta. ¿Y sabías donde querías estudiar?) Me encanta la tecnología (...) entonces quería una universidad fuerte en ese tema, por eso postulé al MIT (Massachusetts Institute of Technology) (...) Me encanta Europa, pero en la tecnología son los Estados Unidos los primeros, es otro mundo. Tal vez hay personas que no están de acuerdo conmigo, pero si quieres estar en la vanguardia de la tecnología, tienes que ir a Estados Unidos".

Los profesionales que han trabajado en el sector privado prefieren estudiar en Estados Unidos. En el caso de Bruno, su decisión se apoya en un contenido en particular, sin embargo, para la mayoría se trata de elegir un título que sea reconocido y prestigioso en el país de origen.

Ahora veamos el relato de Manuela, chilena e ingeniera de la Universidad Católica, hija dos médicos, que decide estudiar un MBA en Boston. Ella financia sus estudios en parte gracias al apoyo de su empleador, una empresa de consultoría financiera de origen estadounidense con sede en Santiago. "Durante mucho tiempo tuve la idea de estudiar en Francia, o más ampliamente en Europa, porque mi marido tiene la nacionalidad francesa. Él no quería estudiar, así que pensábamos que teniendo la nacionalidad no tendría problemas para trabajar mientras yo estudiaba. Incluso tomé clases de francés y todo. Pero todo el mundo me decía que fuéramos mejor a Estados Unidos. Cuando buscaba un profesor para tener una carta de recomendación, todos eran graduados de universidades de Estados Unidos, y todos trataban de convencerme de que yo hiciera lo mismo. Seguro que hay universidades prestigiosas y todo, pero el problema es que la gente en Chile no lo sabe. Además, las universidades francesas están mal posicionadas en los rankings internacionales, y era un requisito para mi empresa postular a programas de las 


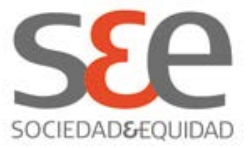

diez mejores universidades del mundo. De lo contrario habría tenido que pagar por todo mi cuenta. Después, mis colegas pensaban lo mismo, que por una cuestión de redes profesionales, era mil veces mejor estudiar en Estados Unidos, pues es inversión que te abre puertas en todas partes. Por último, el tema del idioma, el francés me resultaba difícil, en cambio yo ya hablaba inglés en su momento. Finalmente, por el tema de los rankings y de las redes decidí estudiar en los Estados Unidos". La primacía de este país respecto de Europa es innegable en el entorno académico y laboral de los profesionales que trabajan en las empresas. Por otra parte, el tema del financiamiento es fundamental para ellos debido a que los programas profesionales en Estado Unidos suelen tener un alto costo ${ }^{4}$. La mayor parte de los profesionales como Manuela diversifican las fuentes de financiamiento considerando ahorros, préstamos bancarios y becas de estudio.

La exploración de las trayectorias de profesionales de quienes siguen un postgrado en el extranjero luego de trabajar en las empresas indica que su decisión es una bifurcación activa. Ellos disponen de recursos económicos y simbólicos para partir y se encuentran al mismo tiempo expuestos a un entorno laboral que promueve la internacionalización. Favorecidos con relación a otros, el gatillador de su partida puede encontrarse en el deseo de ascender profesionalmente y dejar un trabajo poco estimulante, o bien en el plano personal, considerando que muchos de estos entrevistados son solteros. En cualquier caso, hacer un postgrado afuera resulta relativamente fácil para ellos, aunque al mismo tiempo se trata de personas que no estaban necesariamente predispuestas u obligadas a migrar.

Los profesores de educación primaria y secundaria: retomar los estudios para cambiar de trabajo

Esta categoría reúne a un grupo de jóvenes que tienen un título universitario y una experiencia profesional como profesores en establecimientos escolares de su país. Para ellos, el proyecto de estudiar un postgrado en el extranjero constituye una estrategia para alejarse de este tipo de empleo. En el contexto chileno y colombiano, la privatización de los sistemas escolares se vincula con una fuerte precariedad del trabajo de los profesores. Los bajos salarios y la dificultad para obtener un puesto estable, se suman a largas jornadas de trabajo y cursos con más de 30 alumnos. Estas

4 Por ejemplo, el costo anual del programa de MBA del Massachusetts Institute of Technology asciende a 58,200 dólares para el año 2012-2013. A este monto deben sumarse los costos de vida estimados por esta institución alrededor de 40,000 dólares que consideran alojamiento, transporte, libros y comida para una persona en la ciudad de Boston. http:// mitsloan. mit.edu/ mba/ admissions/ financial-aidl/ 


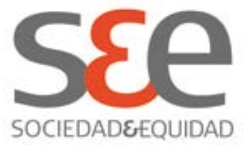

circunstancias permiten entender por qué muchos jóvenes profesores siguen viviendo con sus padres después de varios años de ejercicio profesional. Estos elementos contribuyen al desencanto gradual de algunos maestros. Otros profesionales no están decepcionados sino que creen que la educación continua es parte fundamental de su trabajo. Para todos ellos, el proyecto de estudiar en el extranjero permite aspirar a un cambio en su carrera profesional.

Para ilustrar este análisis, veamos el relato de Arturo estudiante chileno que sigue un máster profesional en París. "(Pregunta: ¿Por qué quisiste seguir estudiando? ¿Qué esperabas con ello?) Pensé en el hecho de que soy profesor, pero no me veo a mí mismo como profe para toda la vida. Estoy convencido de que la diferencia generacional entre una persona cincuenta años y un niño de diez no ayuda a hacer un buen trabajo con él (...). Así que si yo no iba a ser profesor de por vida, tenía que buscar otra cosa en la cual especializarme y reorientar mi carrera. Pero yo no quería cambiar por completo, habría significado tener que hacer otra licenciatura. Y ahí es donde encontré este programa que me pareció muy interesante sobre el envejecimiento y la discapacidad. Soy un profesor de educación diferencial, por lo que pude ver la relación con la discapacidad. El envejecimiento, por otro lado, aportaba el lado nuevo que yo buscaba, que me permitiría encontrar otro trabajo en el futuro no necesariamente en la enseñanza". Cuando la estrategia de estudiar se orienta al mundo profesional, las personas prefieren estudiar un programa de máster.

Otros jóvenes en cambio, quieren trabajar luego en la educación superior. Ellos deciden mayoritariamente hacer un doctorado.

Veamos la historia de Simón, estudiante colombiano de doctorado en Nueva York. "Cuando usted es un graduado en historia, no tiene demasiadas oportunidades de trabajo. O bien trabaja en un colegio como profesor, o si no empieza una carrera en la investigación. Por mi parte, la enseñanza no me interesaba demasiado. Así que decidí hacer una tesis para así trabajar en las universidades después". Las disciplinas estudiadas por estos jóvenes están orientadas hacia la investigación y la enseñanza. En nuestra muestra se trata de profesionales que han cursado un pregrado en idiomas, ciencias sociales, artes 0 literatura.

Una de las características del entorno de trabajo de los profesores es el acceso a las oportunidades institucionales de perfeccionamiento. Muchas de ellas consideran oportunidades para estudiar en el extranjero ${ }^{5}$. Estas

5 La formación de profesores parece ser una prioridad. Ello refleja los esfuerzos de los países por invertir en la sociedad del conocimiento y buscar efectos de replica costeando los estudios de postgrado de los docentes. A través de las entrevistas recolectamos información detallada sobre algunas instituciones que disponen de 


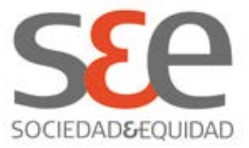

alternativas pueden satisfacer su deseo de un cambio y de ascensión profesional y social que se instala entre los profesores jóvenes. Veamos el relato de Rayen, chilena y profesora de danza que parte a estudiar a Francia sin tener una situación financiera estable. "Cuando me decidí a partir, vendí mi auto para venir a París y ver in situ lo que podía estudiar. Tiempo después me gané la beca de la Embajada (programa de becas Ile-de-France), sin eso no habría podido nunca venirme y pagar mis estudios (...) Muchas personas que conozco tienen problemas para renovar sus permisos de residencia. Nunca ha sido mi caso, tengo la suerte de poder decir que lo que quieren escuchar en la Prefectura. Que soy becaria del gobierno francés, que me matriculé en la Facultad de Medicina de París V y que además soy profesora". El profesor que está perfeccionándose es una de las figuras privilegiadas de la movilidad internacional.

En este grupo, las profesionales cuentan con un capital económico, cultural y social diverso. Más de un tercio de sus padres son también profesores. Algunos pueden ser considerados como pertenecientes a las clases medias bajas y no disponían de antemano de los recursos financieros y simbólicos necesarios para emigrar.

Veamos el caso de Jacqueline, colombiana graduada en educación diferencial. Su historia muestra cómo la influencia de la cooperación internacional le permite preparar un doctorado en neurociencias en Estados Unidos. Jacqueline es hija de una mujer sin estudios y jefa de familia. Ella vive con su madre y su hermana en la ciudad de Manizales hasta la edad de 34 años cuando obtiene una beca de estudios. "Siempre quise aprender Inglés. Con el primer sueldo que tuve, tomé clases en el Instituto norteamericano de mi ciudad (...) Mientras estaba trabajando, un colega me aconsejó que enviara mi hoja de vida al colegio norteamericano, ya que siempre estaban buscando maestros bilingües. Yo no tenía todavía un buen nivel, pero como iba a organizando principalmente talleres con los padres, me contrataron de todas formas (...) En este colegio, nuestro nivel de inglés era medido continuamente por el Instituto Norteamericano. La señora de la recepción me decía todo el tiempo 'Usted tiene el nivel para postular al programa Fulbright' (...) Yo quería especializarme en neurología, pero no había suficientes alternativas en Manizales en esta área (...). Un día pensé bah por qué no ir, vamos a averiguar qué hay que hacer para estudiar en Estados Unidos. Pero no tenía ni idea de cómo buscar programas de estudio ni nada. Cuando estás ahí, sientes que es

programas de becas de estudios en el extranjero para profesionales de la educación: los gobiernos (CONICYT en Chile y el Ministerio de Educación Nacional en Colombia), el sistema DAAD de cooperación alemana, el programa Fulbright en Estados Unidos y el Banco Mundial. 


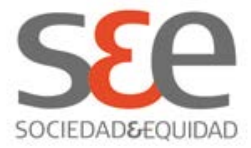

imposible siquiera imaginar estudiar en otro país. En este sentido, el Instituto Norteamericano me ayudó mucho. La señora de la recepción que ya me conocía, era la responsable del departamento de orientación. Ella me explicó cómo ponerme en contacto con las universidades y lo que tenía que hacer para enviar mi postulación a Fulbrigth". Los sistemas institucionales que buscan favorecer la movilidad de estudiantes tienen una influencia decisiva para este tipo de trayectorias profesionales.

La decisión de hacer un postgrado en el extranjero puede ser considerada en este grupo como una bifurcación de tipo "concurso afortunado de circunstancias" de acuerdo a la tipología bifurcaciones de Valentine Hélardot. Esta noción indica la acción de un contexto favorable a los proyectos y deseos del individuo. En este sentido, seguir un postgrado en el exterior aparece, para en el caso de los jóvenes profesores, como el resultado de programas de becas y el deseo de estos profesionales de enfrentar las dificultades asociadas al trabajo en los sistemas escolares.

Los profesionales insatisfechos: a la búsqueda de un título para encontrar un trabajo estable o realizar una reconversión profesional.

Después de haber terminado sus estudios de pregrado, algunos jóvenes encuentran dificultades para insertarse en el mundo laboral porque no logran encontrar rápidamente un primer trabajo, o bien porque los puestos a los que acceden no cumplen con sus expectativas. Estas circunstancias son un resultado inesperado y doloroso considerando los esfuerzos individuales y familiares para obtener su título universitario. En este contexto, los entrevistados ofrecen explicaciones diversas que dan cuenta de su frustración y de una sensación de injusticia. Veamos por ejemplo el relato de Antonia, arquitecta chilena hija de padres con estudios superiores, quien al momento de la entrevista sigue un doctorado en París. "En mi profesión, los estudios de arquitectura se aprovechan de los recién graduados, les pagan muy mal y los obligan a trabajar hasta tarde sin tener ningún tipo de seguridad laboral. Yo trabajaba mucho, demasiado, y aunque fui la mejor de mi promoción, no pude conseguir ni un contrato decente en varios años". El relato de Santiago, licenciado chileno sociología, también sirve para ilustrar este tipo de trayectoria. "No sé por qué no tuve un buen trabajo antes de venirme a Francia. Otros compañeros de mi curso empezaron pegas (trabajos) estables y con buena plata, pero yo no. Siempre estuve haciendo pequeños proyectos, donde mi trabajo era valorado, pero una vez terminado, no había otra cosa. Y vuelta a pasar un mes buscando trabajo terminar en otro proyecto corto". La categoría analítica que llamamos los profesionales insatisfechos reúne primero algunas disciplinas de estudios que se caracterizan por proporcionar inserción laboral incierta. En nuestra muestra se trata de sociólogos, algunos economistas, diseñadores y arquitectos, 


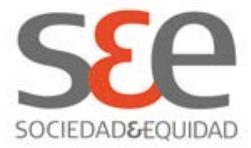

pero también podríamos pensar en otras disciplinas como periodismo o psicología. Algunos de estos profesionales los encontramos en otras categorías, como en los trabajadores del sector público y de las universidades. Sin embargo, otros no logran conseguir con facilidad un empleo que les corresponda. Esta heterogeneidad de circunstancias tiene probablemente relación con la baja regulación de los sistemas de educación superior de los países estudiados. El elevado número de universidades que ofrecen programas de educación en estas disciplinas no toma en cuenta las oportunidades concretas a las cuales sus titulados podrán luego acceder ${ }^{6}$.

Los factores agregados o estructurales no explican por sí solos por qué algunos jóvenes profesionales están satisfechos antes de partir a estudiar al extranjero. En esta categoría, también consideramos las personas decepcionadas de su profesión un punto de vista personal. Veamos el caso de Mateo chileno y graduado en Sociología. "Cuando terminé la universidad, no tuve problemas para encontrar un trabajo en un centro de investigación de la Facultad de Ingeniería de la Universidad Católica. Trabajé allí durante varios años (...) Estaba contento de tener trabajo, pero no era lo mío, no me gustaba. Pensé que tal vez era por esta pega en particular, rodeado de ingenieros, así es que traté de cambiarme, pero daba pésimas entrevistas, no tenía la motivación correcta (...) Sufrí ene, pero un día, finalmente me di cuenta de que la sociología no era para mí". Mateo decide dejar su trabajo y postula a un máster en cinematografía. Como sabía francés y su novia también, decidieron juntos retomar sus estudios en Francia.

En último término, esta categoría considera también a profesionales universitarios que ejercen empleos inestables y mal remunerados solicitando de su parte un fuerte compromiso personal. Estas personas describen su trabajo como una pasión lo que les permite relativizar las condiciones de trabajo. En nuestra muestra, encontramos dos casos. El primero se trata de profesionales que trabajan en causas sociales, como es el caso de Nelson y Paulina, un ingeniero y una economista colombianos. Ambos trabajaban proyectos comunitarios descritos como "muy interesantes" pero que no les permiten ganar lo suficiente para vivir. En segundo lugar, pensamos en las personas que trabajan en el dominio de la música o el arte. Estos profesionales trabajan en un medio particular, donde parecen disfrutar de un ambiente bohemio o de

6 Este desajuste se ve agravado debido al bajo control de las estrategias de algunas instituciones privadas de educación superior. Los programas de estudios mencionados continúan creciendo en matrícula debido a que son carreras rentables para las universidades. Estas instituciones las ofrecen sin tener que realizar una inversión importante en investigación, infraestructura o tecnología, saturando de esta manera el mercado de trabajo de estas profesiones (Espinoza, 2005: 49). 


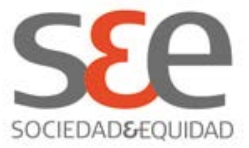

creación lo que oculta muchas veces salarios bajos e inestabilidad. La literatura sobre la precariedad de los trabajadores calificados puede ser útil para comprender mejor estos casos. Por ejemplo, la investigadora Rosalind Grill (2012) pone de relieve la precariedad existente en el trabajo creativo de artistas, productores, periodistas y otros profesionales.

Para todos ellos, la idea de estudiar en el extranjero parece ser una buena alternativa para mejorar su futuro profesional. Algunos piensan que a través la especialización será posible aspirar a un trabajo estable con un mejor salario. Este es el discurso sencillo y práctico de Beatriz, diseñadora que estudia un máster en Paris: "Quise hacer un postgrado pensando que un título adicional podría abrirme otras puertas a nivel profesional". Para aquellos que quieren cambiar de trabajo como Mateo, estudiar afuera es la oportunidad de reconvertirse haciendo otros estudios más cercanos a sus deseos. Este es también el caso de Paulina, quien no admite que nunca quiso estudiar economía. "Elegí esa carrera sobre todo para complacer a mis padres, pero yo siempre quise estudiar Historia".

Si estas personas han decidido reanudar sus estudios, ¿por qué no intentarlo en su propio país? ¿Por qué estudiar en el extranjero? Estos jóvenes dan cuenta de un interés particular por el extranjero que se relaciona con su socialización. Por ejemplo, la mitad de los entrevistados de este grupo son egresados de la escuela francesa en Santiago. Otros se refieren a su familia u otras personas significativas que les inculcaron la idea de viajar. También podemos pensar en un efecto de generación: algunos indican haber sido influenciados en sus decisiones por la partida de otros amigos durante este período de insatisfacción profesional. En este sentido, el caso de Chile para también relacionarse con el aumento significativo del número de becas de estudio para postgrado en el extranjero (más de la mitad de los entrevistados chilenos de la muestra son becarios).

La preparación del proyecto de estudios en el extranjero es facilitado por el período relativamente corto entre la graduación y salida al exterior. Estos jóvenes reciben ayuda de sus antiguos profesores de la universidad para preparar sus solicitudes y postulaciones.

La totalidad de este grupo decide partir estudiar a Francia. Para las personas que no obtienen una beca y que debido a su trayectoria no cuentan tampoco con ahorros, el costo más bajo de los programas de estudio en las universidades francesas, así como la posibilidad de trabajar en ese país, son factores cruciales que pesan en la elección del país de estudio. Sin lugar a dudas para ellos su decisión puede ser considerada como una bifurcación activa. En cambio, respecto de aquellos que realizaron sus estudios en el colegio la Alianza Francesa, el conocimiento del sistema de educación francés y su 


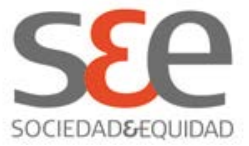

relación con los programas específicos de cooperación internacional resultan claramente una ventaja. En este sentido podríamos decir que su decisión es menos una bifurcación dado que existe una continuidad fuerte en el plano cultural y simbólico. Por último, los jóvenes graduados en ciencias sociales se inclinan frecuentemente hacia Francia considerando el prestigio de ciertos autores y escuelas en estas disciplinas en las universidades latinoamericanas.

\section{Conclusión}

En el ámbito profesional, hacer un postgrado en el extranjero es una práctica favorable a los deseos individuales. Para algunos, es la oportunidad para construirse como el dueño de su propio destino, casi como una figura mítica, y de desafiar ciertas dificultades como la precariedad salarial, el desempleo o el desencanto profesional, desde una posición activa y rupturista. Para otros en cambio, estudiar fuera del país refleja una capacidad particular de adaptación y de negociación que les permite orquestar dificultades y oportunidades en un entorno laboral cambiante, globalizado y cada vez más exigente.

Sin embargo, el análisis de la dimensión profesional constituye sólo una lectura posible de estas trayectorias migratorias. Desde la perspectiva de la socialización de clases, un postgrado en el extranjero aparece como una ausencia de bifurcación para los jóvenes que pertenencen a las clases altas. El título de postgrado internacional es una herramienta eficaz para confirmar su elevada posición social y escapar a la amenaza que representan nuevos grupos sociales en ascenso. Por esta razón, estudiar en el extranjero es una práctica altamente estructurada y no espontánea para los miembros de las elites económicas y políticas. Una suerte de "corredor" se impone a ellos de manera que mayoritariamente eligen sólo ciertos programas de estudios en algunas instituciones específicas, como por ejemplo, la formación de ingenieros o de policy-makers en las universidades de Harvard o Chicago. Además, su situación de pareja y familiar debe mostrar coherencia y sumisión a los valores tradicionales asociados a estos grupos.

Otro ejemplo de ausencia de bifurcación puede leerse en las trayectorias que llamamos "internacionalizadas". Estos itinerarios forman parte de las clases medias favorecidas o ascendentes, pero su principal característica no es la condición de clase sino una socialización sostenida e intensa basada en referencias internacionales. Para estos jóvenes, la idea de estudiar un postgrado en el extranjero es una continuidad, y probablemente, esta experiencia no será tampoco su última estancia en el extranjero.

Los entrevistados que pertenecen a las clases menos favorecidas de la muestra, siguen una dirección opuesta a aquéllos que pertenecen a las élites. Las dificultades económicas se ven agravadas a veces por los episodios de 


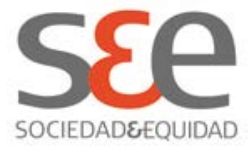

discriminación social, lo que no impide sin embargo múltiples logros académicos y profesionales. El período de estudios en el extranjero se basa en la esperanza de ganar una herramienta basada en el mérito, el título internacional, que podrá reforzar su búsqueda de trabajo. Para algunos, esta búsqueda de reconocimiento no excluye el comienzo de nuevos proyectos migratorios más permanentes.

Por último, para otros jóvenes profesionales, la decisión de perfeccionarse en Francia o en Estados Unidos, puede ser considerada como evento poco previsible. Aún cuando estos individuos forman parte de las clases medias acomodadas, lo que valdría suponer una emigración más probable, sus lógicas de acción son particulares e irreductibles. Estas experiencias se alejan del modelo de ultra-estructurado de las élites y de las disciplinadas búsquedas de prestigio de aquellos profesionales con padres sin estudios superiores. La oportunidad de obtener rápidamente una beca de estudios o el hecho de movilizar ciertos recursos sociales y simbólicos, les permite emigrar y posponer así una situación profesional poco consolidada. Sus decisiones no aparecen relatadas como un proyecto o una estrategia precisa, sino como una respuesta del momento, elegida entre otras alternativas disponibles.

Las trayectorias menos previsibles de los estudiantes de postgrado permiten comprender ciertos espacios de azar y, por qué no, de libertad y de cambio subjetivo desde el punto de vista del individuo. Por otra parte, el contexto de dichas trayectorias tiene que ver con el crecimiento progresivo de los estratos medios en las sociedades latinoamericanas, y con los efectos de las inversiones en capital humano avanzado. Ambos enfoques contribuyen a explicar la diversificación de los perfiles de los migrantes altamente calificados.

\section{Referencias Bibliográficas}

Agulhon C. y Xavier De Brito A. (2009). Les étudiants étrangers à Paris. Entre affiliation et repli. Paris: L'Harmattan.

Bolzman C. y Guissé I. (2010). La formation des étudiants. Réalités et obstacles à la mobilité internationale dans les rapports Nord-Sud. In : A. MANÇO et C. BOLZMAN. Transnationalités et développement. Paris: L'Harmattan, p. 141154.

Cortes G. y Faret L. (2009). Les circulations transnationales, lire les turbulences migratoires contemporaines. Paris : Armand Colin.

De Coninck F. y Godard F. (1990). L'approche biographique à l'épreuve de I'interprétation. Revue française de sociologie, 31, p. 23-53. 


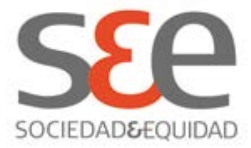

Espinoza O. (2005). Privatización y Comercialización de la Educación Superior en Chile: una visión crítica. Revista de la Educación Superior, 34 (3), Numero 135, Julio-Septiembre, p. 41-60.

Grill R. (2012). De nouvelles classes sociales émergent-elles à partir du travail créatif ? Communication dans la journée d'études: Les travailleurs intellectuels à l'épreuve des transformations de l'emploi et du capitalisme. Nouvelles figures, nouvelles classes ? EHESS, 22 octobre, Paris.

Hélardot V. (2010). Vouloir ce qui arrive ? Les bifurcations biographiques entre logiques structurelles et choix individuel. In : Marc Bessin, Claire Bidart et Michel Grossetti : Bifurcations. p. 160-167. Paris : La Découverte.

Meyer J.- B. (2008). La mobilité des compétences dans une société mondiale basée sur les savoirs. Habilitation à diriger des recherches sous la direction de Bertrand Badie. Paris: Presses de la Fondation Nationale des Sciences Politiques.

Sassen S. (2001). The Global City: New York, London, Tokyo. Princeton: Princeton University Press.

Schnapper D. (1999). La compréhension sociologique. Démarche de l'analyse typologique. Paris: Presses Universitaires de France.

Wihtol De Wenden C. (2010). La question migratoire au XXle siècle. Paris: Presses de la Fondation Nationale des Sciences Politiques. 